
\title{
R Research Square \\ Challenges in antibiotics prescription in pediatrics: A national survey among Lebanese pediatricians
}

\section{Amale ISSA}

Saint Joseph University

Nadine SALEH

Lebanese University

Mira HLEYHEL

Lebanese University

Rouba Karen ZEIDAN

Lebanese University

Sara ASSAF

Lebanese University

Nathalie LAHOUD ( $\nabla$ nathalie.lahoud@hotmail.com )

Lebanese University

\section{Research Article}

Keywords: antibiotic prescribing, attitude, practice, pediatricians.

Posted Date: March 17th, 2021

DOI: https://doi.org/10.21203/rs.3.rs-334293/v1

License: (c) (i) This work is licensed under a Creative Commons Attribution 4.0 International License.

Read Full License 


\section{Abstract \\ Background}

Antibiotics are the most prescribed drugs especially in pediatrics and for Upper Respiratory tract Infections (URI). Physicians are facing many challenges in their practice regarding antibiotics prescription. The aim of this study, the first of its kind in Lebanon, is to evaluate attitudes and practices of Lebanese pediatricians towards antibiotics prescription with a special focus on URI in order to identify challenges facing physicians.

\section{Methods}

This is a cross-sectional study conducted in 2018 among pediatricians registered in the Orders of Physicians. A 63-item questionnaire in English was sent by e-mail or by message to the mobile phone of all pediatricians with subsequent reminders. Data was collected from May 1st through July 2nd 2018. Statistical analysis was done using SPSS 21.0. The analysis involved descriptive quantitative statistics (means and standard deviations, frequencies and percentages).

\section{Results}

From a total of 1213 physicians approached, 117 agreed to participate in the study giving a response rate of $\sim 10 \%$. A high proportion of Lebanese pediatricians replied correctly on avoiding antibiotics in cases of cough (86.3\%), URI (74.4\%), tympanic membrane dysfunction (64.1\%) and prevention of secondary infection (63\%). Eighty percent of physicians prescribed antibiotics for pharyngitis without requesting a throat culture. The majority of physicians admitted that antibiotic use is considerable in their community and that antibiotic resistance is a threat on the national and international level. Forty percent of pediatricians agreed and strongly agreed that giving advice to parents reduces their antibiotic demand. The same percentage disapproved that they might prescribe antibiotics in order to gain parents' trust.

\section{Conclusion}

National campaigns are required to increase parents' education and promote judicious antimicrobials use. Implementing national guidelines, ensuring continuing medical education for doctors and regulating over the counter sale of antibiotics are highly recommended.

\section{Introduction}

Antibiotics are the most prescribed drugs especially in pediatrics (1). In 2002-2010, a study done in the USA concerning drug prescription trends in children found that the most commonly used medication classes were antibiotics (2). Upper Respiratory Tract Infections (URI) are among the most frequent 
infections for which antibiotics are prescribed in pediatrics (3). Those infections are the first cause for parents to consult primary care physicians (4). Most of the URI are caused by viruses (5), hence they are self-limited and does not require antibiotic administration except in limited indications (6). It is well cited in the literature that inappropriate antibiotic prescription for URI is common in ambulatory settings, and as many as 10 million antibiotic prescriptions per year are directed toward respiratory conditions for which they are unlikely to provide benefit (7).

According to the World Health Organization (WHO), antibiotic resistance is an important public health issue (8). Several factors have been shown to promote the development of antimicrobial resistance; most importantly is medical misuse of antibiotics defined by prescribing antibiotics when not indicated or for an inappropriate duration or the prescription of broad-spectrum antibiotics when more selective ones would have been sufficient (9-10). More than half of all community-based antibiotic prescriptions may have been unnecessary or inappropriate due to patient, physician and health system factors (11).

Physicians are facing many challenges throughout their daily practice in antibiotic prescription such as parental pressure (12), diagnostic uncertainty (13), drug promotion (12) and over the counter (OTC) sale of antimicrobials (14). Several studies have explored the knowledge, attitudes, and practices of pediatricians concerning antibiotic prescriptions and evaluated the factors associated with those prescriptions $(12,15-18)$. Many qualitative studies were conducted about this subject in inpatient and ambulatory pediatrics settings $(12,19)$. But few quantitative studies were carried out in the outpatient settings concerning antibiotic prescription in URI (20).

In Lebanon, a developing country in the Middle East, there are no national guidelines regarding antibiotics prescription for children with URI. Furthermore, the Lebanese Ministry of Public Health (MOPH) considers antimicrobial resistance a serious threat according to a statement in 2015 (21). Despite the presence of a law regulating antibiotic prescription in Beirut (22), a previous pilot study resulted in a rate of $42 \%$ of antibiotic dispensing without a medical prescription (23). In addition to that, a high proportion of Lebanese people assumed that antibiotics are used for treatment of common cold and sore throat symptoms (59\%) or viral infections (53\%) (24). Parents thought that antibiotics are effective against viruses $(19.2 \%)$ and both viruses and bacteria (42.6\%). More than half of them never minded to give antibiotics to their child without a physician's prescription (58.4\%) and blamed physicians for antibiotic misuse (52.5\%) (25).

In this context, we conducted this study among the Lebanese pediatricians regarding their attitudes and practices towards antibiotic prescription with a special focus on URI. To the extent of our knowledge, this is the very first national study about this subject in Lebanon.

\section{Methods}

\subsection{Study design and sampling:}


A cross-sectional study was conducted in 2018 among pediatricians across the country. Our target population was limited to pediatricians practicing in Lebanon and registered in the Lebanese Order of Physicians (LOP) of Beirut and Tripoli. A minimum sample of $n=146$ was calculated based on a confidence level of $95 \%$, a margin of error of $5 \%$, a power of $80 \%$, an anticipated proportion of $50 \%$ to allow to maximum sample size and $\mathrm{N}=1316$, the number of registered pediatricians in both orders by March 2018. Unfortunately, the lists provided by the Orders of Physicians had a lot of missing data; we had to search manually the Internet to have more e-mail addresses and phone numbers. We didn't find any contact information for 103 physicians.

\subsection{Study questionnaire and data collection:}

The questionnaire included 63 questions adapted from two previous studies (20) (Appendix A). It was divided into 6 sections asking about: sources of knowledge, 7 clinical scenarios, factors that may influence antibiotic prescribing, the attitude of physicians, interventions to promote prudent use of antibiotics and socio-demographic data.

A pilot study among 10 pediatricians was done before the survey. After the pilot study, we reformulated some questions for means of comprehension and we merged some repetitive questions from the third and fourth section.

The survey was administered in English to all registered pediatricians through an Internet-based questionnaire conducted using Google Forms. It was sent by e-mail or by a message including the link to the online survey to the mobile phone numbers in case no e-mail was provided. A total of 4 e-mails and 2 messages reminders were sent 10 days apart to increase the response rate. Data collection was conducted from May 1st 2018 through July 2nd 2018.

\subsection{Statistical analysis:}

Data analysis was done using Statistical Package for Social Sciences (SPSS) version 21. For the descriptive analysis, continuous variables were presented by means and standard deviations and categorical variables by frequencies and percentages.

\subsection{Ethical considerations:}

Participants read and approved informed consent before completing the questionnaire. Participation was voluntary and physicians were able to withdraw from the survey at any time. Confidentiality and anonymity were respected throughout the study. The ethical committee of the Lebanese University waived the need for an ethical approval taking into consideration the study's objectives and methods.

\section{Results}

\subsection{Socio-demographic characteristics of participants:}


Out of the 1213 physicians approached, 117 agreed to participate in this study giving a response rate of $\sim 10 \%$. Socio-demographic characteristics are shown in Appendix B (Table B 1). Fifty-three percent of physicians were female with a mean $( \pm S D)$ age of $44.5( \pm 12.2)$ years. Fifty-nine percent have been practicing for more than 10 years; around forty percent treated less than 50 patients per week and almost sixty percent had an academic position. Twenty-six percent graduated as specialists in pediatrics from the American University of Beirut and 22.2\% from universities abroad Lebanon. More than half of pediatricians (54.7\%) worked in an urban region only. The majority of participants worked in different governorates and different settings at the same time. Only nine physicians had not attended a continuing medical education event (CME) concerning antibiotics in the past three years.

\subsection{Usefulness of sources of knowledge in the treatment of URI:}

As shown in Table 1. Lebanese pediatricians considered guidelines, previous clinical experience and CME courses very important sources of information in the treatment of URI $(68.4 \%, 71.8 \%, 55.6 \%)$ and important $(29 \%, 26.5 \%, 39.3 \%)$ respectively. Forty-four percent of physicians rated documentation and courses provided by the pharmaceutical industry useful.

\subsection{Self-reported antibiotic prescribing practice of pediatricians:}

The majority of pediatricians responded properly on giving antibiotics for pneumonia (97.4\%) and for cough with nasal discharge (79.5\%) (cases 4 and 7). Avoiding antibiotic prescription was predominant in cases for tympanic membrane dysfunction, cough and URI (cases 1, 2 and 3) with the following percentages respectively: $64.1 \%, 86.3 \%$ and $74.4 \%$. Sixty-three percent of physicians never prescribed antibiotics for the prevention of secondary infection (case 5 ). In contrast, $80.3 \%$ prescribed antibiotics for pharyngitis without ordering a throat culture (case 6) (Table 2).

\subsection{Factors influencing antibiotic prescribing for URI:}

Factors that contributed in an increase in prescription patterns were: preventing serious complications $(65 \%)$, second visit for the same problem (50.4\%), purulent discharge (37.6\%), diagnostic (35\%) or treatment uncertainty (26.5\%). Treatment guidelines decreased antibiotics prescription by only $55.6 \%$. Most of the physicians thought that the child's return to school, parents' requests and expectations, drug promotion, parents' satisfaction, fever, purulent nasal discharge and receiving an antibiotic for similar symptoms did not change their practice with frequencies ranging from 60 to $94 \%$ (Table 3).

\subsection{Attitudes of pediatricians:}

Around forty percent of the physicians stated they strongly agree and agree that advising parents reduces their expectations for antibiotics and that prior antibiotic use increases the personal risk of developing resistance $(46.2 \%, 41.9 \% ; 47.9 \%, 41.9 \%$ respectively). Answers from pediatricians with respect to antibiotic use as being a notable factor in their community were as follows: agree $(52.1 \%)$ and strongly 
agree (41.9\%). Physicians strongly agreed and agreed that they prescribe antibiotics when there is a clear indication $(66.7 \%, 29.1 \%)$. Sixty-two percent of physicians were confident that antibiotics are necessary when prescribed. Three-quarters of pediatricians agreed that rapid and effective diagnostic techniques are required for the diagnosis of URI ( $54.7 \%$ agreed and $21.4 \%$ strongly agreed). Most of the doctors agreed and strongly agreed $(54.7 \%, 23.1 \%)$ that parents are convinced that antibiotics should be prescribed for cold and flu symptoms. Physicians believed that if parents feel their child in need of antibiotics then they would manage to obtain antimicrobials from a pharmacy without a prescription (45.3\% agreed and $25.6 \%$ strongly agreed). Opinions were divided among prescribing antibiotics when there is doubt in the bacterial etiology: $35 \%$ of pediatricians approved, $30.8 \%$ disapproved and $26.5 \%$ were neutral. The use of wide spectrum antibiotics in case of doubt in diagnosis or etiology of URI was refuted by $45.3 \%$ and $17.9 \%$ who disagreed and strongly disagreed respectively. As for prescribing antibiotics where it is impossible to conduct a systematic follow up, $43.6 \%$ of pediatricians were adherent and $31.6 \%$ disagreed. Forty percent of physicians disapproved when asked whether they would prescribe antibiotics to gain parents' trust. Pediatricians were against prescribing antibiotics if not indicated because of lack of time ( $65 \%$ strongly disagreed and $29.1 \%$ disagreed). Most of the physicians did not consent that it is difficult for them to withhold antibiotics for cold and flu symptoms because other clinicians prescribe antibiotics for these illnesses (47\% strongly disagreed and $35 \%$ disagreed). Pediatricians highly supported and approved the use of amoxicillin + clavulanic acid more than amoxicillin alone for treating most URI (44.4\%, 24.8\%; 43.6\%, $13.7 \%$ respectively). Antibiotic resistance is considered as a worldwide problem, on both the national and the community level $(53 \%, 53.8 \%, 48.7 \%$ strongly agreed and $41 \%$, 38.5\%, 41\% agreed respectively) (Appendix C: Table C 1).

\subsection{Promoting prudent use of antibiotics in pediatrics:}

When asked about what would aid the prudent use of antibiotics in pediatrics on the national level around $80 \%$ of physicians answered that antibiotics should be dispensed only with a medical prescription; parents' education and physicians CME were also cited. Sixty-one percent considered that media campaigns promoting judicious use of antibiotics should be done. (Fig. 1).

\section{Discussion}

This study showed that the majority of Lebanese pediatricians (97.4\%) replied correctly on prescribing antibiotics for pneumonia (case 4) and avoiding antibiotics in cases of cough (86.3\%), URI (74.4\%), tympanic membrane dysfunction (64.1\%) and prevention of secondary infection (63\%). Eighty percent of physicians prescribed antibiotics for pharyngitis without requesting a throat culture. Practitioners stated that the following factors played a major role in increasing their prescription patterns: prevention of serious complications (65\%), second visit for the same problem (50.4\%), purulent discharge (37.6\%), diagnostic (35\%) or treatment uncertainty $(26.5 \%)$. Most of the physicians considered that parents' requests and expectations (81.2\%), drug promotion (94\%), parents' satisfaction (87.2\%) and fever (72.6\%) did not change their practice. Treatment guidelines were considered to decrease antibiotics prescription by $55.6 \%$. Concerning attitudes of physicians towards antibiotics prescribing the majority of physicians 
admitted that antibiotic use is notable in their community and that antibiotic resistance is a threat on the national and international level. Forty percent of pediatricians agreed and strongly agreed that advising parents reduces their antibiotic demand. The same percentage disapproved that antibiotics prescriptions might be made to gain parents' trust.

In comparison to Canadians doctors, Lebanese colleagues used antibiotics more appropriately in both cases of tympanic membrane dysfunction and cough from sinusitis (first and last cases) (64.1\% versus $43.5 \%, 79.5 \%$ versus $24.5 \%$ respectively). Answers to cases of URI, lobar pneumonia and pharyngitis (cases 3, 4 and 6) were almost identical. As for treating cough and prevention of secondary infections (cases 2 and 5), Lebanese pediatricians prescribed more inappropriate antibiotics in these cases (86.3\% versus $94 \%, 62.4 \%$ versus $82 \%$ respectively) (20). These findings suggest that Lebanese pediatricians had an acceptable use of antibiotics but need more training to improve their practice especially regarding management of pharyngitis and antibiotic prophylaxis.

Several factors influence practitioners' decisions in prescribing antibiotics, Lebanese physicians are in favor of prescribing antibiotics due to the presence of purulent discharge and fever, but less than their Indian colleagues as shown in previous studies (37.6\% compared to $65 \%-88.6 \%$ for discharge, $24.8 \%$ versus 40\%-72.5\% for fever) (26). Diagnostic or treatment uncertainty could increase antibiotic prescription for $35 \%$ and $26.5 \%$ of physicians respectively, compared to $59 \%$ and $53.3 \%$ Indian practitioners respectively (27). Our findings are in line with other studies proving that physicians prescribed antibiotics when they are not certain about the diagnosis (12). Developing clinical tools or laboratory tests can, therefore, aid physicians in making the correct diagnosis. Guidelines in Lebanon were found to decrease antibiotics prescription more than in India (55.6\% compared to 40.6\%). Preventing serious complications increased antibiotics prescription in $65 \%$ of Lebanese physicians versus $79 \%$ of Indians (27). Half of the Lebanese clinicians increased antimicrobials prescriptions if it is the second visit for the same problem confirming that this factor is related to overprescribing (19). Most Lebanese practitioners believed that parents' requests and expectations, drug promotion and parent satisfaction did not change their practice more than Indians physicians (81.2\%, 94\%, 87.2\% compared to 57.2\%, 60.7\% and $64.2 \%$ respectively) (27). Thankfully, pharmaceutical companies' promotional campaigns did not have an influence on Lebanese pediatricians by a greater rate when compared to their Indians colleagues (94\% and 57\% respectively) (26). These findings contradict the studies conducted by Lucas at al. and Rezal et al. who found that clinicians reported prescribing antibiotics due to pressure exercised by drug companies $(12,13)$.

All in all, the percentage of Lebanese pediatricians who increased their antibiotic prescriptions due to several factors was lower than their international colleagues, which is promising and confirms once again that our physicians have a good level of practice.

When it comes to pediatricians' opinions about antibiotics, most of them admitted that antibiotic use is a notable factor in their community but beyond what was found by in Canada (94\% compared to $77 \%$ ); maybe this problem is more critical in developing countries (20). Similarly, the majority stated that 
antibiotic resistance is a concern on the national and international level as stated by the MOPH (21) and WHO (8). Eighty-eight percent of Lebanese physicians are convinced that educating parents will control their demand for antibiotics compared to $93.5 \%$ of Canadian physicians (20). Lebanese doctors believed more than their Canadian colleagues that prior antibiotic use increases the risk of developing resistance (89.9\% compared to $71.8 \%$ ) (20). This confirms that physicians are aware of the principles of antibiotic resistance. Moreover, about three-quarters of Lebanese practitioners acknowledged that parents believe that they should prescribe antibiotics for flu and cold symptoms, which points out the public misconceptions about antibiotics that need to be corrected. Seven pediatricians out of 10 recognized that if a parent feels that his child needs antibiotics he would manage to obtain antimicrobials from a pharmacy without a prescription indicating OTC sale of antibiotics. Almost half of the pediatricians agreed that they would prescribe antibiotics if it were difficult to conduct a follow up echoing overprescribing due to non-clinical factors. This is not an indication by itself for antibiotics, and parents should be educated on how to observe the child and to recognize symptoms that need a follow up consultation. Amoxicillin/clavulanic acid is considered to be the first choice for most URI even more than amoxicillin; while guidelines agree that amoxicillin is the first line treatment (28). Six out of ten physicians were against prescribing wide spectrum antibiotics in case of doubt in diagnosis or etiology of URI, $88.5 \%$ disapproved that they might prescribe antibiotics so that parents continue to trust them. This emphasizes once again that Lebanese clinicians are cautious when prescribing antibiotics. The majority of pediatricians strongly disagreed on prescribing antibiotics if not indicated because of time pressure. This differs from other studies where lack of time during consultation was an incentive to prescribe antibiotics (19) and highlights judicious use of antibiotics in more than half of pediatricians.

\subsection{Strengths and limitations:}

Primarily the innovativeness of this study is a major strength. To the extent of our knowledge, this is the very first study about this subject in Lebanon and the Middle East region. Second, the study included physicians from different regions across the country, graduated from different universities and practicing in different settings and was not limited to one geographic area or to university hospitals. Moreover, administering the survey online has led to complete anonymity, which contributes to less information bias.

As for the study limitations, a selection bias may be present due to missing and non-accurate data in physicians' contact list provided by the Order of Physicians. Furthermore, we didn't find a previously validated questionnaire, but the questionnaire used was based on two previous studies. Third, the response rate was low making generalizability very difficult and the minimal sample size needed being not reached. Unfortunately, not all Lebanese physicians are interested in research participation. Finally, practices of physicians were evaluated based on self-reported practices to clinical scenarios instead of the actual practice; hence there will be an information bias. This might lead to an underestimation of the prevalence of inappropriate attitudes and prescribing behaviors. In fact, prescribing a medication is a multifaceted behavior and it is extremely difficult to measure in real practice unless we conduct a patientsimulated study and the latter might have some ethical considerations to take into account. 


\section{Conclusion}

This study identifies the practice pattern of pediatricians and their attitudes regarding antibiotic usage. Implementation of national guidelines regulating antibiotic prescribing in Lebanon is urgent as well as ensuring CME for doctors concerning antibiotic use. Law enforcement regarding restricting selling OTC antibiotics must be applied in order to decrease antibiotics self-medication. Media especially social media can also play a role in spreading awareness to the public about prudent antibiotics use.

Further studies focusing on pediatricians' antibiotics prescription practices or targeting other primary care physicians are highly praised.

\section{Declarations}

\section{Conflict of interest:}

None.

\section{References}

1. Clavenna A, Bonati M. Differences in antibiotic prescribing in paediatric outpatients. Arch Dis Child. 2011 Jun;96(6):590-5.

2. Chai G, Governale L, McMahon AW, Trinidad JP, Staffa J, Murphy Trends of outpatient prescription drug utilization in US children, 2002-2010. Pediatrics. 2012;130(1):23-31

3. Sellam A et al. Impact of guidelines on ambulatory pediatric antibiotic prescriptions. Arch Pediatr Organe Off Soc Francaise Pediatr. 2015 Jun;22(6):595-601.

4. McCormick A et al. Morbidity statistics from general practice. Fourth national study 1991-1992. London: HMSO, 2006.

5. Pediatric Treatment Recommendations | Community | Antibiotic Use | CDC [Internet]. 2017 [cited 2017 Dec 21]. Available from: https://www.cdc.gov/antibiotic-use/community/for-hcp/outpatienthcp/pediatric-treatment-rec.html

6. Earnshaw, S., Monnet, D. L., Duncan, B., O'Toole, J., Ekdahl, K., Goossens, et al. European Antibiotic Awareness Day, 2008 - the first Europe-wide public information campaign on prudent antibiotic use: methods and survey of activities in participating countries. Euro surveillance : European communicable disease bulletin, 14(30), 19280.

7. Hersh AL, Shapiro DJ, Pavia AT, Shah SS. Antibiotic prescribing in ambulatory pediatrics in the United States. Pediatrics. 2011; 128(6):1053-1061 2.

8. Leung E et al. The WHO policy package to combat antimicrobial resistance. Bull World Health Organ. 2011 May 1;89(5):390-2. 
9. Holmes AH, Moore LSP, Sundsfjord A, Steinbakk M, Regmi S, Karkey A, et al. Understanding the mechanisms and drivers of antimicrobial resistance. The Lancet. 2016 Jan 9;387(10014):176-87.

10. National Institute on Drug Abuse. Misuse of Prescription Drugs 2020 [online]. Available at https://www.drugabuse.gov/publications/research-reports/misuse-prescription-drugs (accessed on March 1, 2021)

11. Wiskirchen DE et al. Antibiotic stewardship: the FP's role. J Fam Pract. 2016;65(12):876-885.

12. Lucas PJ et al. A systematic review of parent and clinician views and perceptions that influence prescribing decisions in relation to acute childhood infections in primary care. Scand J Prim Health Care. 2015 Mar;33(1):11-20.

13. Md Rezal RS et al. Physicians' knowledge, perceptions and behaviour towards antibiotic prescribing: a systematic review of the literature. Expert Rev Anti Infect Ther. 2015 May;13(5):665-80.

14. Andritsou F et al. Out-of-Hospital Administration of Medication without Prescription and Associated Factors among Preschool Children. BioMed Res Int. 2017;2017:1-9.

15. Faizullah M, Rahman N, Umar Ml, Anwar M, Sarfraz M. A crosssectional study on knowledge, attitude and practices of medical doctors towards antibiotic prescribing patterns and resistance in Khyber Pakhtun Khawah, Pakistan. J App Pharm Sci, 2017; 7 (12): 038-046.

16. Ye D, Yan K, Zhang H, Liu S, Yang C, Jiang M, et al. A survey of knowledge, attitudes and practices concerning antibiotic prescription for upper respiratory tract infections among pediatricians in 2018 in Shaanxi Province, China. null. 2020 Sep 1;18(9):927-36.

17. Lin L, Sun R, Yao T, Zhou X, Harbarth S. Factors influencing inappropriate use of antibiotics in outpatient and community settings in China: a mixed-methods systematic review. BMJ Global Health. 2020 Nov 1;5(11):e003599.

18. Thakolkaran $\mathrm{N}$ et al. Antibiotic prescribing knowledge, attitudes, and practice among physicians in teaching hospitals in South India. J Fam Med Prim Care. 2017;6(3):526-32.

19. Horwood $\mathrm{J}$ et al. Primary care clinician antibiotic prescribing decisions in consultations for children with RTIs: a qualitative interview study. Br J Gen Pract. 2016 Mar;66(644):e207-13.

20. Paluck E et al. Prescribing practices and attitudes toward giving children antibiotics. Can Fam Physician. 2001;47(3):521-527.

21. Ministry of Public Health (MOPH) and the World Health Organization (WHO). Epi-monitor: Antimicrobial resistance. Volume 2, Issue 2- February 2015 [Internet]. [cited 2017 Dec 27]. Available from: http://www.opl.org.Ib/newdesign/PDF/Media/NO-2-2-\%20Antimicrobial-resistance-February2015.pdf

22. OPLI Laws and Regulations [Internet]. [cited 2018 Jan 4]. Available from: http://www.opl.org.lb/newdesign/lawsandregulations.php

23. Cheaito L, Azizi S, Saleh N, Salameh P. Assessment of self-medication in population buying antibiotics in pharmacies: a pilot study from Beirut and its suburbs. Int J Public Health. 2014 Apr;59(2):319-27. 
24. Khalifeh $\mathrm{M}$ et al. Public Knowledge and Attitude towards Antibiotic Use in Lebanon. American Journal of Epidemiology and Infectious Disease, 2017; 5(2), 35-41.

25. Zahreddine $L$ et al. Knowledge of pharmacists and parents towards antibiotic use in pediatrics: a cross-sectional study in Lebanon. Pharm Pract. 2018 Sep 30;16(3):1194.

26. Sivagnanam $G$ et al. A Survey on Current Attitude of Practicing Physicians Upon Usage of Antimicrobial Agents in Southern Part of India. Medscape Gen Med [Internet]. 2004 May 12 [cited 2017 Dec 6];6(2). Available from: https://www.ncbi.nlm.nih.gov/pmc/articles/PMC1395775/

27. Thakolkaran $\mathrm{N}$ et al. Antibiotic prescribing knowledge, attitudes, and practice among physicians in teaching hospitals in South India. J Fam Med Prim Care. 2017;6(3):526-32.

28. Cohen et al. Antimicrobial treatment of ENT infections. Archives de Pédiatrie. 2016;23:S10-6.

\section{Tables}

Table 1: Usefulness of sources of knowledge in the treatment of Upper Respiratory tract Infection:

\begin{tabular}{|c|c|c|c|c|c|}
\hline & $\begin{array}{c}\text { Very } \\
\text { important } n \\
(\%)\end{array}$ & $\begin{array}{l}\text { Importantn } \\
\text { (\%) }\end{array}$ & $\begin{array}{l}\text { Neutral } \\
\mathrm{n}(\%)\end{array}$ & $\begin{array}{c}\text { Not } \\
\text { important } n \\
(\%)\end{array}$ & $\begin{array}{l}\text { Not } \\
\text { important } \\
\text { at all } \\
\quad \mathrm{n}(\%)\end{array}$ \\
\hline Clinical practice guidelines* & $80(68.4)$ & $34(29)$ & $2(1.7)$ & $0(0)$ & $0(0)$ \\
\hline Documentation furnished by the Pharmaceutical Industry & $15(12.8)$ & $52(44.4)$ & $\begin{array}{c}36 \\
(30.8)\end{array}$ & $10(8.5)$ & $4(3.4)$ \\
\hline Courses held by the Pharmaceutical Industry & $14(12)$ & $51(43.6)$ & $\begin{array}{c}35 \\
(29.9)\end{array}$ & $11(9.4)$ & $6(5.1)$ \\
\hline Previous clinical experience & $65(55.6)$ & $46(39.3)$ & $4(3.4)$ & $1(0.9)$ & $1(0.9)$ \\
\hline Continuing Education Courses & $84(71.8)$ & $31(26.5)$ & $1(0.9)$ & $1(0.9)$ & $0(0)$ \\
\hline $\begin{array}{l}\text { Others, e.g., contribution of specialists (microbiologists, } \\
\text { infectious disease specialists...) }\end{array}$ & $57(48.7)$ & $51(43.6)$ & $9(7.7)$ & $0(0)$ & $0(0)$ \\
\hline Contribution of peers (of the same specialization) & $27(23.1)$ & $62(53)$ & $\begin{array}{c}28 \\
(23.9)\end{array}$ & $0(0)$ & $0(0)$ \\
\hline Data collected via the Internet & $12(10.3)$ & $53(45.3)$ & $\begin{array}{c}38 \\
(32.5)\end{array}$ & $12(10.3)$ & $2(1.7)$ \\
\hline
\end{tabular}

* Missing data: 1 (0.9\%)

Table 2: Self-reported antibiotic prescribing practice of pediatricians: 


\begin{tabular}{lcc}
\hline & Correct answers n (\%) & Wrong answers n (\%) \\
\hline Case 1 & $75(64.1)$ & $42(35.9)$ \\
Case 2 & $101(86.3)$ & $16(13.7)$ \\
\hline Case 3 & $87(74.4)$ & $30(25.6)$ \\
\hline Case 4 & $114(97.4)$ & $3(2.6)$ \\
\hline Case 5* & $73(62.4)$ & $42(35.9)$ \\
\hline Case 6 & $23(19.7)$ & $94(80.3)$ \\
\hline Case $7^{* *}$ & $93(79.5)$ & $21(17.9)$ \\
\hline
\end{tabular}

* Missing data: 2 (1.7\%)

** Missing data: 3 (2.6\%)

Table 3: Factors influencing antibiotic prescribing for Upper Respiratory tract Infection:

\begin{tabular}{|c|c|c|c|}
\hline & $\begin{array}{c}\text { Increase antibiotics } \\
\text { prescription } \\
\mathrm{n}(\%)\end{array}$ & $\begin{array}{c}\text { Decrease antibiotics } \\
\text { prescription } \\
\mathrm{n}(\%)\end{array}$ & $\begin{array}{c}\text { No } \\
\text { change } \\
\text { n (\%) }\end{array}$ \\
\hline Productive cough with purulent sputum & $44(37.6)$ & $4(3.4)$ & $69(59.0)$ \\
\hline Second visit for the same problem & $59(50.4)$ & $2(1.7)$ & $56(47.9)$ \\
\hline Fever $\left(>38^{\circ} \mathrm{C}\right)$ & $29(24.8)$ & $3(2.6)$ & $85(72.6)$ \\
\hline Purulent nasal discharge & $32(27.4)$ & $9(7.7)$ & $76(65.0)$ \\
\hline $\begin{array}{l}\text { Patient had received an antibiotic for similar symptoms } \\
\text { in the past }\end{array}$ & $29(24.8)$ & $11(9.4)$ & $77(65.8)$ \\
\hline Patient needs to return to school* & $11(9.4)$ & $2(1.7)$ & $103(88)$ \\
\hline Parents request/expectation & $20(17.1)$ & $2(1.7)$ & $95(81.2)$ \\
\hline Diagnostic uncertainty & $41(35.0)$ & $16(13.7)$ & $60(51.3)$ \\
\hline Parent satisfaction & $10(8.5)$ & $5(4.3)$ & $\begin{array}{c}102 \\
(87.2)\end{array}$ \\
\hline Treatment uncertainty* & $31(26.5)$ & $14(12.0)$ & $71(60.7)$ \\
\hline Treatment guidelines* & $20(17.1)$ & $65(55.6)$ & $31(26.5)$ \\
\hline Drug promotion & $4(3.4)$ & $3(2.6)$ & $\begin{array}{c}110 \\
(94.0)\end{array}$ \\
\hline To prevent serious complications & $76(65.0)$ & $2(1.7)$ & $39(33.3)$ \\
\hline
\end{tabular}

* Missing data: 1 (0.9\%)

Figures 


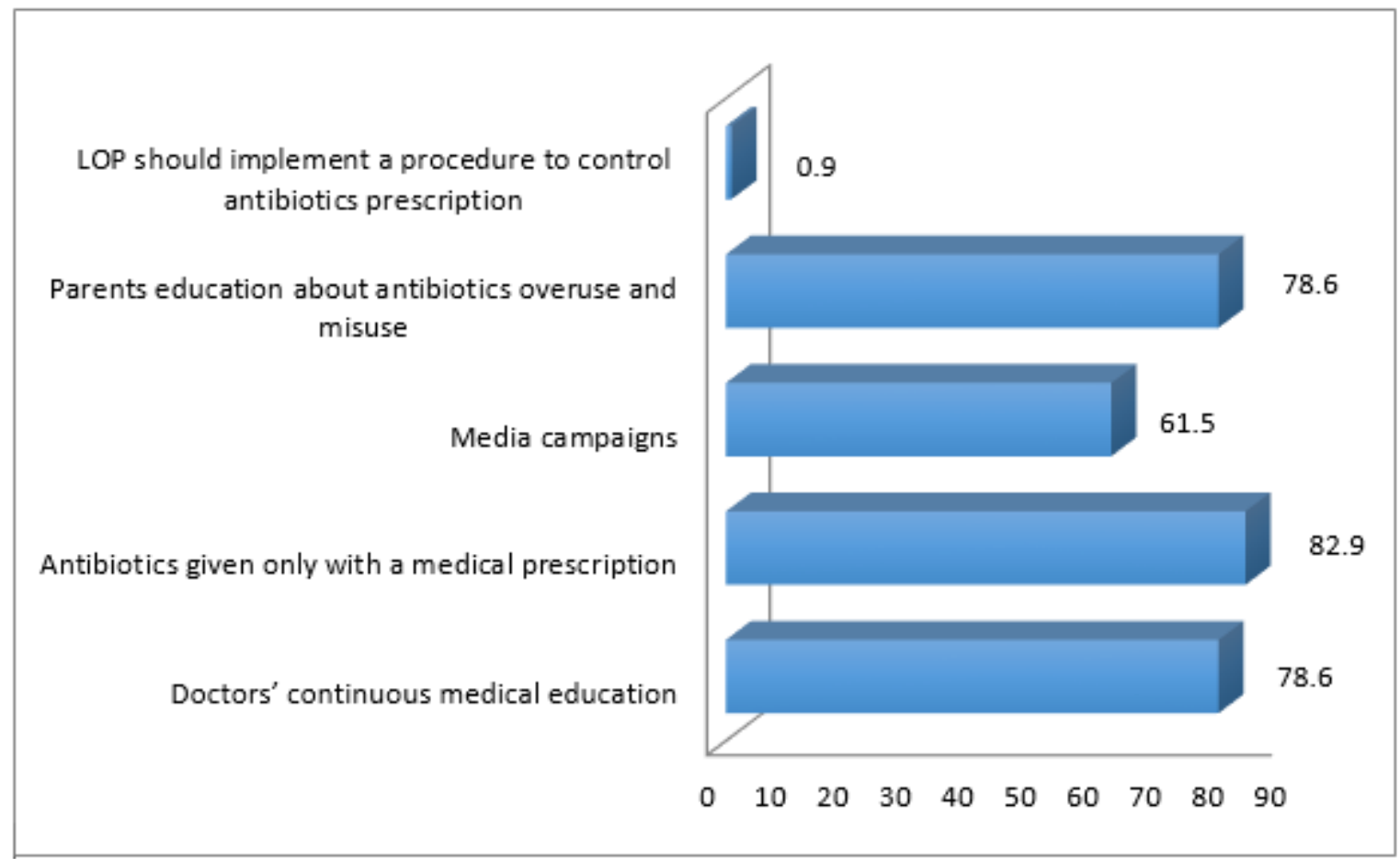

Figure 1: Interventions to promote prudent use of antibiotics in pediatrics

\section{Figure 1}

Interventions to promote prudent use of antibiotics in pediatrics 